\title{
A Novel Time-Resolved Fluorescence Microscope System Extended from the Deep UV-Visible to NIR (240nm - 1700nm)
}

\author{
Lin Chandler, Molecular and Microanalysis Division, Horiba Jobin Yvon, 3880 Park Ave., \\ Edison, NJ 08820, USA
}

Time-resolved fluorescence microscopy is the ultimate tool for investigating dynamic events within cellular/sub-cellular structures and nanomaterials. Applications previously have been limited to wavelengths in the visible region (370-850nm) because microscope optics does not transmit in the deep UV and the detector responds only up to $850 \mathrm{~nm}$. Some of the important applications beyond this visible region cannot be addressed, such as NIR time-resolved measurements from NIR quantum dots $(\mathrm{PbS})$ and intrinsic fluorescence measurements directly from tissue samples. This paper describes a filter-based confocal system (the DynaMyc ${ }^{\mathrm{TM}}$ ) to measure fluorescence lifetimes and intensities directly under the microscope in the wavelength range of 240-1700 $\mathrm{nm}$ for the first time. The system features time-correlated single photon counting (TCSPC) for sensitive and rapid acquisition of fluorescence lifetimes from 100ps to $100 \mu \mathrm{s}$. An Olympus BX51 microscope was modified to transmit light for both excitation and emission down to 240nm in the deep UV. To maintain maximum light throughput, both the excitation light source and the detector are directly coupled to the microscope. Excitations are fast pulsed solid state LEDs or laser diodes. The detectors are the fast response TBX detector for UV-VIS (240-850nm) and the Hamamatsu H10330 PMT for NIR (950-1700nm). This system can be used to obtain fluorescence lifetime and intensity map with variable spatial resolution $(>1 \mu \mathrm{m})$ with an automated stage. This paper will present fluorescence lifetime measurement from a single protein crystal with a diameter of $70 \mu \mathrm{m}$, and lifetime mapping obtained from a FRET tissue slide. The preliminary data for NIR lifetimes measured from NIR quantum dots $(\mathrm{PbS})$ and solar cells on silicon wafer will be presented. The potential application for detection of intrinsic fluorescence from single proteins in the deep UV and deep tissue imaging in the NIR will also be discussed. 